\title{
Relationship of Arrhythmogenic Substrate and Frequent Ventricular Extrasystoles in Patients with Nonischemic Cardiomyopathy
}

Cristiano de Oliveira Dietrich ${ }^{1, *}$

ORCID IDS

Dietrich CO (DD https://orcid.org/0000-0002-7373-9119

\begin{abstract}
Cardiomyopathies (CMP) are associated with fibrosis scarring and myocardial fiber disarray that promotes slow tissue conduction and substrate for reentrant ventricular arrhythmias. Sometimes CMP may be associated with ventricular extrasystoles, but they may originate infrequently in the scar tissue. The case report presents a patient with nonischemic CMP, frequent premature ventricular ectopias and sustained ventricular tachycardia referenced to catheter ablation.
\end{abstract}

KEYWORDS: Premature ventricular contraction; Ventricular tachycardia ablation; Cardiomyopathy. 


\section{INTRODUCTION}

Frequent premature ventricular complexes (PVCs) can induce a cardiomyopathy that may be reversible with catheter ablation ${ }^{1}$. However, patients with frequent PVCs also have an increased risk of mortality, which may be attributable to concomitant heart disease ${ }^{2}$. Patients with ischemic cardiomyopathy and remote myocardial infarction present PVCs most often originate at low-voltage areas ${ }^{3}$. Postinfarction PVCs often have origin related to the exit site of a reentrant $\mathrm{VT}^{4}$. Therefore, PVCs ablation is associated with the loss of inducible ventricular tachycardia ${ }^{4}$. In patients with frequent PVCs and nonischemic cardiomyopathy, the arrhythmogenic substrate was located in scar tissue ${ }^{5}$. The case report shows a patient with nonischemic CMP and frequents ventricular arrhythmias submitted to catheter ablation.

\section{PRESENTATION}

An 80-year-old male patient was referred for catheter ablation due to recurrent ventricular tachycardia (VT) and frequent premature ventricular contractions (PVCs) despite anti-arrhythmic therapy (metoprolol and amiodarone). The patient has long-standing persistent atrial fibrillation (AF) and hypertension under therapy with Eliquis, metoprolol and valsartan. Amiodarone was initiated due to sustained VT (Fig. 1) and high PVC burden (15-20\% in 24 h-Holter). Transthoracic echocardiogram showed a normal left ventricle (LV) ejection fraction (EF), and late enhanced MRI, which demonstrated a normal LVEF (51\%) and fibrosis in the anterobasal and anteroseptal LV segments. After the 3rd episode of VT, the patient was submitted to catheter ablation.

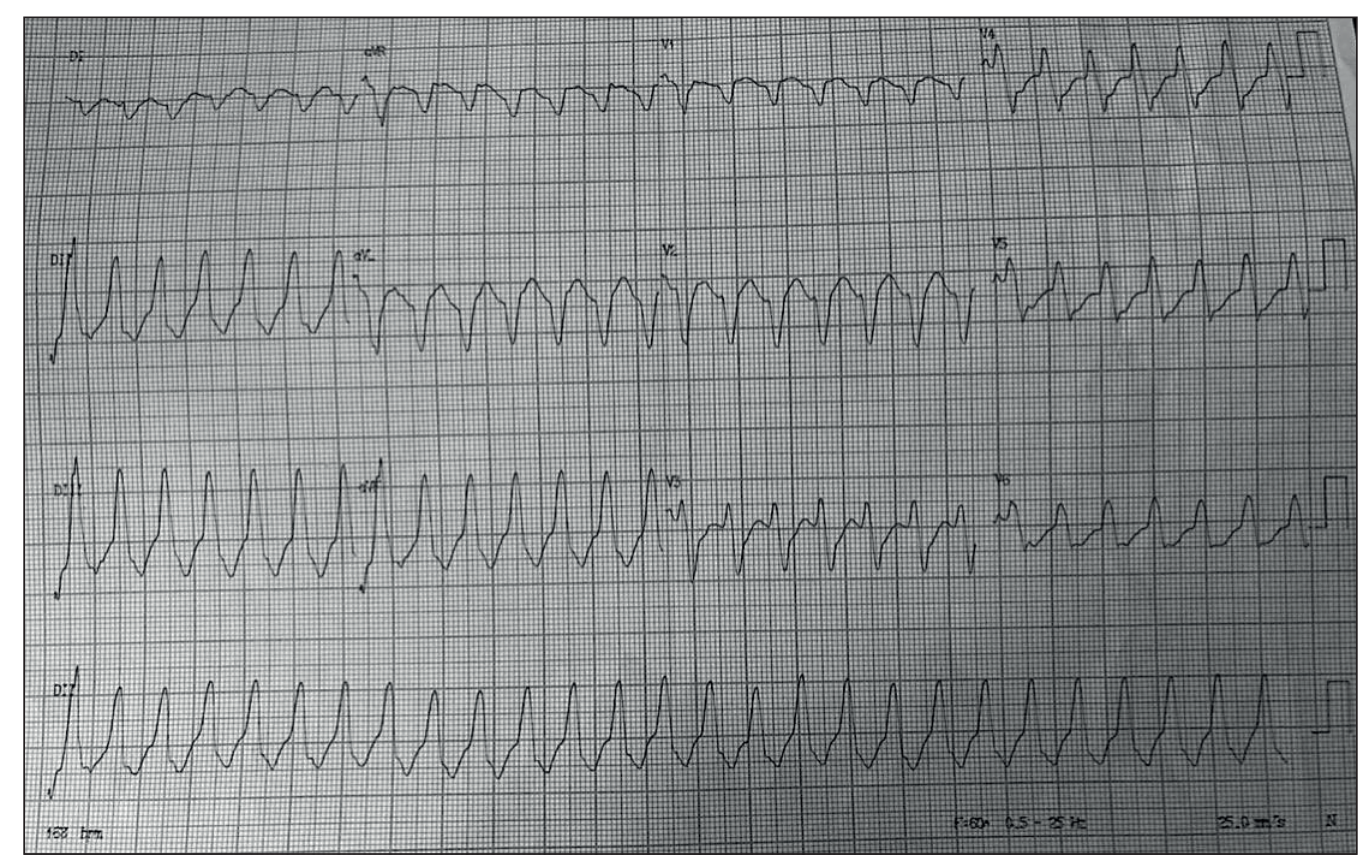

Figure 1. Clinical ventricular tachycardia.

Electrophysiologic study was performed under general anesthesia and post-absorptive state. Briefly, two femoral vein punctures underwent to insert a diagnostic decapolar catheter inside the coronary sinus and intracardiac echocardiography catheter (View Flex, Abbott) to acquire cardiac image. Baseline rhythm was AF, frequent PVCs with two ECG morphologies (Figs. 2a and 3a) and nonsustained monomorphic VT (up to 3 beats). His bundle recording showed a normal HV interval (55 ms). Three-dimensional (3D) geometry of LV and right ventricle (RV) were performed using EnSite Precision system and HD Grid catheter. Then, activation mapping of both PVCs found the source of origin by the earliest activated area: 
1) first PVC (LBBB pattern): septal between posterior right ventricular outflow tract (RVOT) and right coronary cusp (RCC) source (Fig. 2); and 2) second PVC (RBBB pattern): anterobasal LV just beneath aortic valve source (Figs. 3).

Pace-mapping performed in these precocity areas resembled the PVCs as a good match (>10/12), as can be seen in Figs. $2 \mathrm{~B}$ and $3 \mathrm{E}$ (red arrow). Baseline rhythm voltage mapping showed a small scar (bipolar electrograms $<0.5 \mathrm{mV}$ ) in anterobasal LV with recording of late potentials (LP) that represented a deceleration zone by isochronal late activation mapping (ILAM), (Fig. 3, See Supplementary Material). Radiofrequency energy was delivery in earliest area of RVOT to
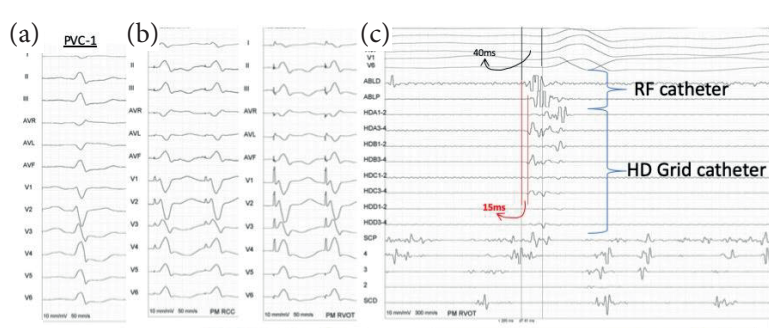

Note:

Pace-mapping presents better match in right coronary cusp (RCC) than posteroseptal RVOT - B; however, RVOT was activated earlier than RCC as showed
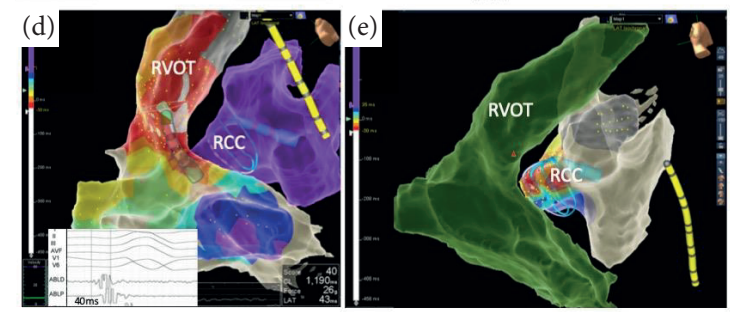
by electrogram precocity $(\mathrm{C})$ and activation mapping (D-E): RVOT 15 ms earlier than RCC. Intracardiac echocardiogram (ICE) images (F and $\mathrm{G}$ ) show the catheter positioned in RCC (HD grid) and RVOT (RF catheter) during recoring of electrograms $(\mathrm{C})$.
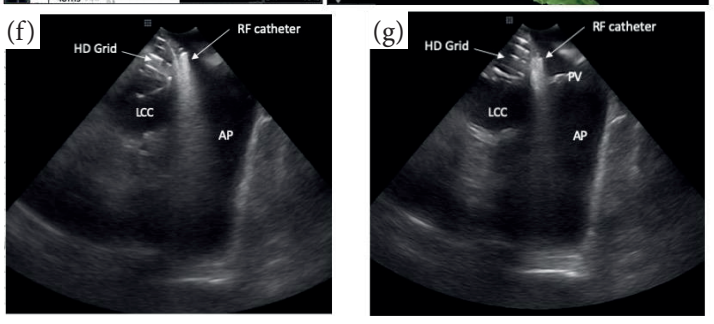

LCC $=$ left coronary cusp

RCC $=$ LCC $=$ left coronary cusp

$\mathrm{PV}=$ pulmonary valve

$\mathrm{AP}=$ pulmonary artery

RVOT $=$ right ventricular outflow tract

Figure 2. Mapping (b to g) and ECG (a) of premature ventricular contractions (PVC) with LBBB pattern.

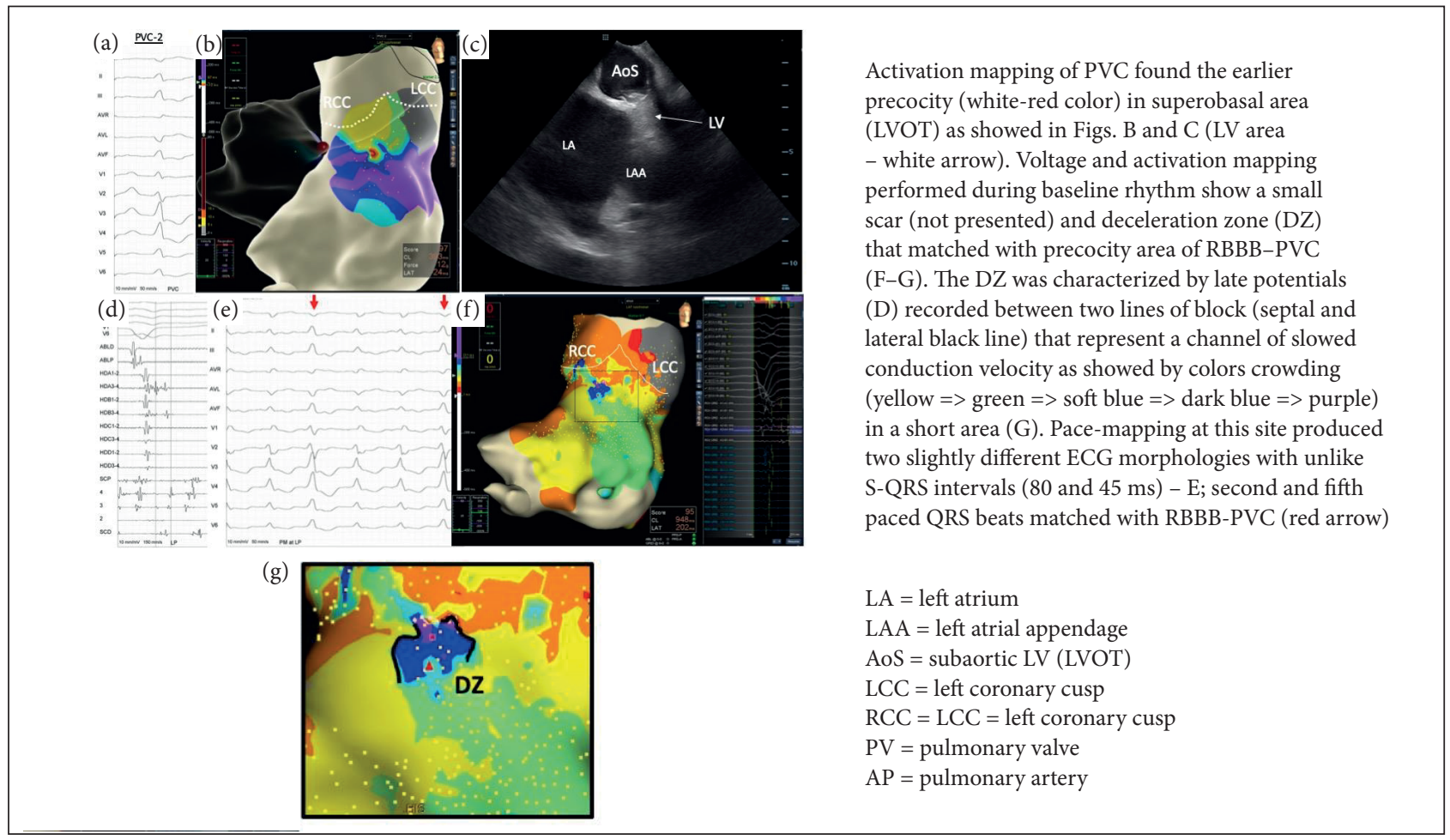

Figure 3. Premature ventricular contractions (RBBB pattern) and arrhythmogenic substrate of left ventricle (LV) 
eliminate the first PVC (LBBB pattern). After, another RF energy was delivered in the channel defined as DZ of the ILAM that was sufficient to abolish the second PVC (RBBB pattern) and all late potentials recorded in the scarring myocardial area (Fig. 4). After, VT was not induced by programmed ventricular stimulation (up to 3 extra-stimuli).

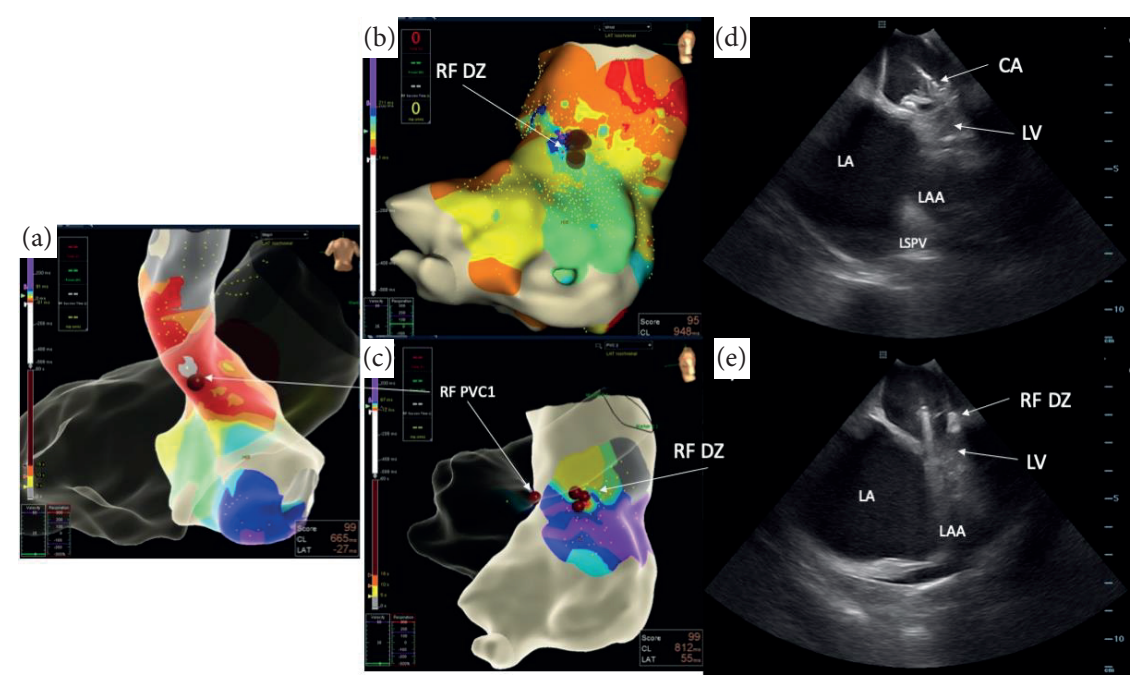

A and C: radiofrequency (RF) energy offer in posteroseptal RVOT eliminated PVC-1 (RF PVC1 arrow). Deceleration zone (DZ) guided the RF delivery to abolish PVC-2 and modify the ventricular arrhythmogenic substrate (DZ RF arrow). Position of the ablation catheter (AC) in the DZ viewed by ICE (D). Also, the RF lesion (RF DZ) after stopped the energy deliver was showed by ICE catheter in the superobasal RV.
LSPV = left superior pulmonary vein

$\mathrm{LA}=$ left atrium

LAA $=$ left atrial appendage

$\mathrm{AP}=$ pulmonary artery

Figure 4. Set of ablation lesions in the arrhythmic sources.

After hospital discharge, no recurrences occurred during three months of follow-up and amiodarone was withdrawal due to previously adverse effect in the thyroid function. No complications after procedure or follow-up were documented.

\section{DISCUSSION}

The mechanisms of PVCs in patients with structural heart disease have included both substrate-based ectopy and idiopathic PVCs unrelated to the underlying cardiomyopathy. In the first hypothesis, the origin of PVCs may be from areas of scarring or abnormal myocardium. However, the idiopathic PVCs from RVOT or LVOT (or other sites) may be found in patients with cardiomyopathy. In this context, the case report describes a patient with nonischemic cardiomyopathy that presents a PVC related to an arrhythmogenic scar placed in superobasal LV, and an idiopathic PVC originated from septal myocardium between RVOT and RCC unrelated to myocardial disease.

Substrate mapping based in the electrograms performed during sinus or paced rhythm help to find a slowed conduction channels that are critical to sustained reentrant VT. Three-dimensional voltage mapping can be useful to show an abnormal area of scarring tissue. Then, 3D activation mapping adjusting the latest signal of electrograms may be used to define the abnormal activated areas within the scar or a low-voltage area. Recently, Aziz et al. reported that ILAM analysis incorporated to a 3D electroanatomical system is a useful tool to demonstrate the critical zone of reentrant circuit ${ }^{6}$. The areas, termed as deceleration zone (DZ), can be visualized by ILAM as colors crowding in a small area. The DZ was strongly related to isthmus of induced VT of patients with different cardiomyopathies. In this case report, the author showed a precise relation between the DZ or substrate mapped during baseline rhythm and the clinical ventricular arrhythmias (VT and PVC). 


\section{CONCLUSION}

This case report showed the characteristic of ventricular arrhythmias potentially presented in patients with nonischemic cardiomyopathy. Idiopathic or substrate related PVCs can be found in this population. Catheter ablation using PVC coupled to the substrate mapping help to understand and decrease recurrences in the follow-up.

\section{REFERENCES}

1. Bogun F, Crawford T, Reich S, Koelling TM, Armstrong W, Good E, et al. Radiofrequency ablation of frequent, idiopathic premature ventricular complexes: Comparison with a control group without intervention. Heart Rhythm. 2007;4(7):863-7. https://doi. org/10.1016/j.hrthm.2007.03.003

2. Dukes JW, Dewland TA, Vittinghoff E, Mandyam MC, Heckbert SR, Siscovick DS, et al. Ventricular ectopy as a predictor of heart failure and death. J Am Coll Cardiol. 2015;66(2):101-9. https://doi.org/10.1016/j.jacc.2015.04.062

3. Sarrazin J-F, Good E, Kuhne M, Oral H, Pelosi F, Chugh A, et al. Mapping and ablation of frequent post-infarction premature ventricular complexes. J Cardiovasc Electrophysiol. 2010;21(9):1002-8. https://doi.org/10.1111/j.1540-8167.2010.01771.x

4. Bogun F, Crawford T, Chalfoun N, Kuhne M, Sarrazin JF, Wells D, et al. Relationship of frequent postinfarction premature ventricular complexes to the reentry circuit of scar-related ventricular tachycardia. Heart Rhythm. 2008;5(3):367-74. https://doi.org/10.1016/j. hrthm.2007.11.026

5. El Kadri M, Yokokawa M, Labounty T, Mueller G, Crawford T, Good E, et al. Effect of ablation of frequent premature ventricular complexes on left ventricular function in patients with nonischemic cardiomyopathy. Heart Rhythm. 2015;12(4):706-13. https://doi. org/10.1016/j.hrthm.2014.12.017

6. Aziz Z, Shatz D, Raiman M, Upadhyay GA, Beaser AD, Besser SA, et al. Targeted ablation of ventricular tachycardia guided by wavefront discontinuities during sinus rhythm: A new functional substrate mapping strategy. Circulation. 2019;140(17):1383-97. https://doi.org/10.1161/CIRCULATIONAHA.119.042423 